\title{
Encoding processes in memory scanning tasks
}

\author{
JEFFREY O. MILLER and ROBERT G. PACHELLA \\ University of Michigan, Ann Arbor, Michigan 48104
}

\begin{abstract}
Three experiments are presented that deal with the effect of stimulus probability on the encoding of both alphanumeric characters and nonsense figures. Experiment I replicated a previous finding of an interaction between stimulus probability and stimulus quality in a memory scanning task with numbers as stimuli. Experiments II and III investigated the same paradigm with unfamiliar visual forms as stimuli, and no interaction was found. Results were interpreted as showing that probability affects the encoding mechanism only when the encoding process results in a representation of the name of the stimulus. When stimulus materials are visual forms without names, probability does not appear to affect encoding processes.
\end{abstract}

An issue that has received great attention recently is the effect of stimulus probability on the memory scannng or character classification task extensively studied jy Sternberg $(1966,1967,1969)$. Theios, Smith, Haviland, Traupmann, and Moy (1973) have shown that stimulus probability affects reaction time (RT) in this task and, on the basis of this result, challenged Sternberg's (1969) four-stage model. Stemberg's (1969) model includes an encoding stage, in which the subject forms an internal representation of the test item; an ixhaustive comparison stage, in which the subject compares the test item against every item in the positive set; a response choice stage, in which the subject exImines an internal switch to see whether or not a match sccurred at any time during the comparison stage; and a response execution stage, in which the subject performs the appropriate response. As Theios et al. (1973) have pointed out, the comparison mechanism in this model does not account for stimulus probability effects, since on each trial the probe item is compared to every positive set item. They proposed a new model in which the comparison stage terminates as soon a a match is found, and both positive and negative set items are compared with the probe or test item. In the Theios et al. (1973) model, the order of comparison is influenced by probabiltiy, with the probe being compared earlier (on the average) with higher probability stimuli. Since the comparison process terminates when a match is found, the comparison process tends to be shorter when a high probability probe is presented, with a corresponding decrease in RT. Thus, this model accounts for the stimulus probability effect by a mechanism in the comparison stage.

This research was supported by the Advanced Research Projects Agency, U.S. Department of Defense, and monitored by the U.S. Air Force Office of Scientific Research under Contract AF44620-72-C-0019 with the University of Michigan, Human Performance Center. Requests for reprints should be sent to Robert G. Pachella, Human Performance Center, 330 Packard Road, Ann Arbor, Michigan 48104.
Certain evidence suggests that it might not be neccessary to explain the stimulus probability effect by means of a mechanism in the comparison stage. Miller and Pachella (1973) attempted to determine in which stage stimulus probability has its effect. They covaried stimulus probability and stimulus quality in a character classification task, and found an interaction between these two factors. They concluded that stimulus quality and stimulus probability must affect some common stage of processing: namely, the encoding stage. This finding suggested that Sternberg's (1969) model might not be incompatible with stimulus probability effects, but posed a problem for the Theios et al. (1973) model, in which the effect of probability was explained in the comparison stage. Furthermore, three studies have directly examined the comparison stage to test for evidence of probability effects there. Klatzky and Smith (1972) varied positive set size and probabilistic expectancy and found no interaction. They concluded that expectancy does not affect the comparison stage. Both Theios and Walter (1974) and Biederman and Stacy (1974) tested for an interaction of stimulus probability and positive set size. While the data from these studies are very similar, Theios and Walter (1974) found the interaction and concluded that probability affects the comparison stage in accordance with the Theios et al. (1973) model, but Biederman and Stacy (1974) found no such interaction and concluded that probability must have its effect in some stage other than the comparison stage. Biederman and Stacy (1974) argued that stimulus probability affects the response choice mechanism.

One problem with the inference that stimulus probability affects encoding arises from the lack of interaction between set size and stimulus quality (Sternberg, 1967; Bracey, 1969). Since set size was confounded with stimulus probability in these experiments, set size and stimulus quality should have interacted. To account for this lack of interaction, it may be necessary to distinguish expectancy effects from number of items effects (e.g., Biederman \& Stacy, 1974). Relative prob- 
ability of items within a set may determine expectancy and thereby influence the encoding process, while the number of items in the memory set may affect only the comparison process.

\section{EXPERIMENT I}

The locus of the stimulus probability effect is of crucial importance in the controversy between the memory scanning models of Sternberg (1969) and Theios et al. (1973). The experiments of Miller and Pachella (1973) indicate that probability has at least part of its effect on the encoding stage, but these experiments have recently been questioned by Biederman and Stacy (1974). They expressed concern that the unusual method of degradation used by Miller and Pachella (1973) may have led to atypical results. That method of degradation involved presenting the stimulus at low contrast initially, and increasing the contrast during the trial. Biederman and Stacy (1974) suggested that this method of degradation might have affected some other stage in addition to the encoding stage. The purpose of Experiment I of the present paper was to replicate Experiment I of Miller and Pachella (1973) using a more conventional method of degradation.

\section{Method}

Apparatus. The stimuli were the digits one-eight presented on a cathode ray tube (CRT) controlled by PDP-1 computer. Subjects sat abcut $65 \mathrm{~cm}$ from the CRT and responded on a microswitch keyboard directly in front of them. Degradation was produced by covering the entire face of the CRT with a sheet of acetate. This sheet reduced the intensity of the stimulus by about $1.5 \log$ units, thus reducing the contrast between stimulus and background.

Subjects and procedure. Subjects were 24 paid volunteers from the University of Michigan campus. All subjects were right-handed. The procedure was nearly identical to that of Experiment I of Miller and Pachella (1973). Subjects were given a set of digits to remember prior to the experiment. One digit was presented on each trial, to which subjects were required to give the positive response if the digit was one of the prevvously memorized set of digits and the negative response otherwise. Each subject was run in four blocks of 200 trials. Positive set was held constant across the four blocks, and blocks alternated between high- and low-contrast stimuli, with the order of high-and low-contrast blocks counterbalanced across subjects. Subjects made the positive response with their right index fingers and the negative response with their left index fingers. Each subject had a positive set of Size 4 and a negative set of Size 4. The four items in each set were assigned probabilities $.275, .125, .075$, and .025 , and these probabilities were held constant throughout the four blocks. Stimulus sequences were generated by a pseudorandom computer algorithm individually for each subject and block, with the constraint that the frequency of each stimulus within a 200-trial block corresponded exactly to its assigned probability. Positive sets were chosen randomly and counterbalanced across subjects, so that each digit occurred equally often at each probability.

The subjects were instructed as to the nature of the task, and were given the positive set. They were also told to be as fast and accurate as possible, but they were not informed of the probability manipulation. At the beginning of each block, the subjects were given a verbal ready signal. Then the fir: stimulus appeared, and remained on the CRT until a respons was made. "Right" or "wrong" feedback was given after eac response, and about $1 \mathrm{sec}$ later the next stimulus appearec

\section{Results and Discussion}

For each block of 200 trials, the average RT an percentage error were computed at each level of stimulu probability for both positive and negative responses These values were averaged across subjects and respons type separately for high- and low-contrast blocks, an the results are shown in Figure 1. An analysis of varianc of RTs yielded significant main effects of Stimulu Contrast $\left[F(1,22)=45.4, p<.01, \mathrm{MS}_{\mathrm{e}}=82,452\right]$ an Stimulus Probability $[F(3,66)=31.5, \quad p<.01$ $\left.\mathrm{MS}_{\mathrm{e}}=13,003\right]$. The interaction of Stimulus Quality b: Stimulus Probability was also significant $[F(3,66)$ : $\left.3.09, \mathrm{p}<.05, \mathrm{MS}_{\mathrm{e}}=7,455\right]$. The only other significan interactions were the interactions of Practice by Qualit. $\left[\mathrm{F}(1,22)=6.38, \mathrm{p}<.01, \mathrm{MS}_{\mathrm{e}}=33,611\right]$ and Practic by Probability $\left[F(3,66)=5.94, p<.01, \mathrm{MS}_{\mathrm{e}}=5,457\right]$ Both the Quality effect and the Probability effec decreased with practice. In the analysis of error rates the only significant effect was that of Probability $\left[F(3,66)=6.96, p<.01, \mathrm{MS}_{\mathrm{e}}=11,429\right]$, with mor errors being made to low-probability stimuli.

The results of this experiment replicate those 0 Miller and Pachella (1973). The Probability effect i very nearly the same in the normal conditions of the two experiments, while the effect of Stimulus Quality wa larger in the earlier experiment because of the differen

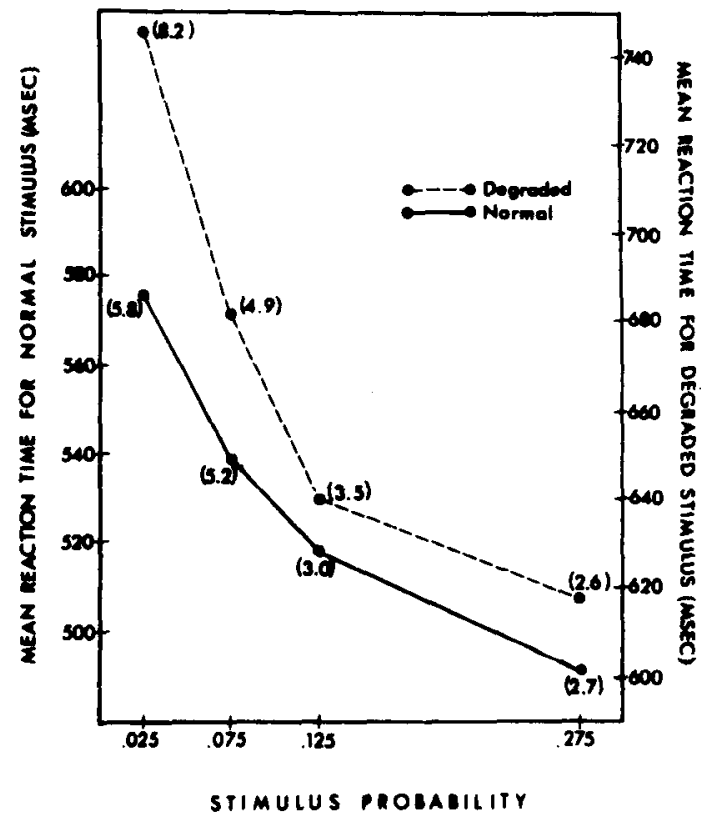

Figure 1. Reaction time as a function of stimulus probability for the normal stimulus and degraded stimulus conditions in Experiment I. (The average percentage error at each point is indicated in parentheses. The scale for the normal stimulus con dition has been shifted upwards.) 
method of degradation. The interaction of Probability by Quality is significant in both studies, though it was larger in the earlier experiment, probably because of the larger effect of Stimulus Quality. In this experiment, as well as the experiments of Miller and Pachella (1973), the effect of degradation increased monotonically as stimulus probability decreased. Although the Probability by Quality interaction is not significant when the lowest probability point is excluded from the analysis, the consistent finding of a monotonic interaction suggests that there may well be an interaction of Probability and Quality at probability levels in the range of .275 to .075 , though a more powerful experiment would be required to detect it statistically. Thus, both experiments support the idea that stimulus probability affects the encoding stage, despite different methods of producing degradation.

\section{EXPERIMENT II}

The evidence is strong that stimulus probability affects the encoding stage in a memory scanning task, so it makes sense to ask how, or through what mechanism, probability affects the encoding process. The mechanisms of the encoding process in RT paradigms have not been studied extensively, but there is evidence that the final output of the encoding process (i.e., the representation of the stimulus used in the comparison stage) is in the form of a name code. ${ }^{1}$ Sternberg (1967) and Bracey (1969) varied memory set size and stimulus quality and found no interaction, at least for practiced subjects. This finding indicates that the comparison process involves a representation of the stimulus which retains no effects of visual degradation. Wattenbarger (Note 1; see also Nickerson, Note 2, for a discussion of Wattenbarger's work) varied the case of the memory set letters and the probe letters in a memory scanning task. One condition required subjects to respond ignoring case. In the other condition, subjects were to make positive responses only if the probe had been given in the positive set in the case in which it was probed. In the latter condition, the subjects were to give negative responses if the probe letter had appeared in the positive set in the other case. The crucial comparison was of comparison rates in these two tasks as compared to a third task in which case was not varied. Wattenbarger reasoned that if the subjects used internal representations of the names of stimuli, then memory scanning rates should be about equal in the task where subjects ignored case and in the task where case was not varied. If the subjects used internal representations of a visual nature, then memory scanning, rates should be about equal in the task where subjects had to respond on the basis of case and in the task where case was not varied. His data supported the notion of name-code representations.

Chase and Calfee (1969) investigated the effects of acoustically and visually confusable memory sets in a memory scanning task. They found that memory searching was slower through an acoustically similar positive set than through visually similar or neutral positive sets. This finding provides further evidence that internal representations used in this task are of an acoustic nature.

Given the evidence that the final form of the encoded stimulus is the name of the stimulus, a rudimentary model of the encoding process can be considered. Theios (1975) has recently developed such a model, and the model includes two distinct mechanisms. The first is an input stage that transforms the physical stimulus into a code that can be used in memory. The second process is an identification stage that transforms the stimulus code into the name code of the stimulus. Presumably, during this stage the stimulus trace makes contact with long-term memory, and the name of the probe is retrieved for the subsequent comparison operations. Stimulus probability might affect either one or both of these substages of the encoding process.

One way to discover where probability has its effect is to remove one of the substages. Consider a memory scanning task with a set of stimuli which do not have associated name codes. The visual preprocessing mechanism should operate in much the same manner for these stimuli, though perhaps more slowly, since one would not expect processing prior to identification to be qualitatively influenced by the type of identification that will result at the end of the process. However, the second stage must obviously be quite different, since no name representation exists for these stimuli. Experiment II investigated the encoding process in a memory scanning task where the stimuli do not have name codes associated with them, in an attempt to delineate the locus of the probability effect within the encoding stage. If probability has a similar effect on the encoding stage in a task where stimuli do not have the name codes, then it is reasonable to infer that probability has its effect on the visual preprocessing substage of encoding. However, a finding that probability has no effect on encoding in such a task would be strong evidence that probability affects the retrieval of a name code for the probe. Note that it would not be valid to predict that, since a substage is deleted when stimuli do not have name codes, RTs should be faster overall than when stimuli do not have name codes. Other stages could be slowed by the use of a different type of code. In particular, Briggs and Swanson (1970) showed that the comparison stage proceeded much more slowly for nonsense forms than for alphanumeric stimuli. It would also not be valid to predict that, if probability does not affect encoding in this task, the overall effect of probability should be smaller than it was in tasks where probability did affect encoding. We do not believe that probability affects only the encoding stage, but rather that it has some of its effect on encoding. Modifying the task in order to remove the effect of probability on encoding could increase the duration 

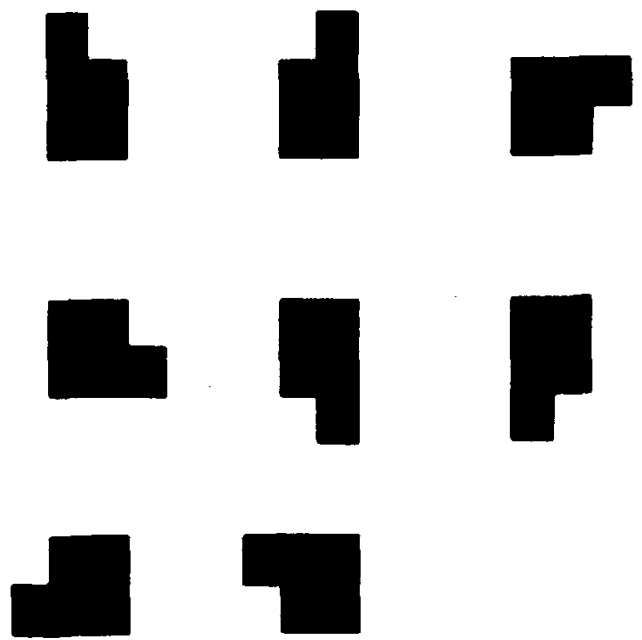

Figure 2. Stimuli used in Experiment II.

of other probability sensitive stages. For example, if probability were to affect the comparison stage, then, on the basis of the work of Briggs and Swanson (1970), probability should have a larger effect on the comparison stage for nonsense forms than for digits. Thus, the effect of probability could be larger for nonsense forms than for digits, even if a probability sensitive processing stage is deleted. The only prediction relative to the present experiment that can be made from the hypothesis that probability affects the retrieval of a name code is that probability and quality should not interact when no name-code retrieval occurs.

\section{Method}

Apparatus. The stimuli are shown in Figure 2. They were presented on a CRT controlled by an IBM 1800 computer. Subjects sat about $65 \mathrm{~cm}$ from the CRT and responded on a microswitch keyboard. Degradation was produced in the same manner as in Experiment I.

Subjects and procedure. Twenty-four right-handed subjects were secruited from the University of Michigan campus. Participation was voluntary, and subjects were paid $\$ 2 / \mathrm{h}$. The procedure was identical to that of Experiment 1 with the following exceptions: (1) Each subject was run in three blocks of 200 trials instead of four. The first block was a practice block so the subjects could learn the stimuli, and it was always run without degradation. The order of degraded and normal contast in the second and third blocks was counterbalanced across subjects. (2) The subject had a third response key which he could press at any time, causing the trial sequence to be interrupted and the positive set to be displayed on his CRT.

\section{Results and Discussion}

Figure 3 shows the average RTs and percentages of error at each level of stimulus probability for both high and low contrast. In the analysis of error rates, only the main effects of Stimulus Quality $[\mathrm{F}(1,22)=8.0$, $\left.\mathrm{p}<.01, \mathrm{MS}_{\mathrm{e}}=36\right]$ and Probability $[\mathrm{F}(3,66)=5.56$, $\left.\mathrm{p}<.01, \mathrm{MS}_{\mathrm{e}}=108\right]$ were significant. In the analysis of variance of RTs, the only significant main effects were Stimulus Quality $\left[\mathrm{F}(1,22)=43.8, \mathrm{p}<.01, \mathrm{MS}_{\mathrm{e}}=\right.$ $28,072]$, Response Type $[F(1,22)=10.6, p<.01$,
$\left.\mathrm{MS}_{\mathrm{e}}=28,687\right]$ and Probability $[\mathrm{F}(3,66)=32.6$. $\left.\mathrm{p}<.01, \mathrm{MS}_{\mathrm{e}}=25,998\right]$. The interaction of Stimulus Quality by Probability, which is shown in Figure 3, was not significant $\left[F(3,66)=.7, M_{e}=5,348\right]$. Since the interaction of Quality by Probability is not significant with these stimulus materials, it is tempting to conclude that the place where Quality and Probability overlap in their effects is the process of arriving at a name code for the stimulus. However, this is a rather strong conclusion to be drawn on the basis of a single experiment which accepted the null hypothesis. Furthermore, the stimulus materials in Experiment II differ from the digits used in Experiment $I$ in other ways besides lacking a verbal code. The figures in Experiment II were solid rather than line figures, and clearly had fewer visual features than the set of digits one-eight. Experiment III is an attempt to replicate Experiment II with a set of stimulus materials visually more similar to digits.

\section{EXPERIMENT III}

\section{Method}

The stimuli for this experiment are shown in Figure 4. They were presented on a CRT controlled by a PDP-1 computer Subjects were run in four blocks of 200 trials, alternating be tween high- and low-contrast blocks. No practice block was given. All other aspects of the method are identical to those in Experiment II.

\section{Results and Discussion}

The average RTs and percentages of error at each level of stimulus probability and quality are shown in

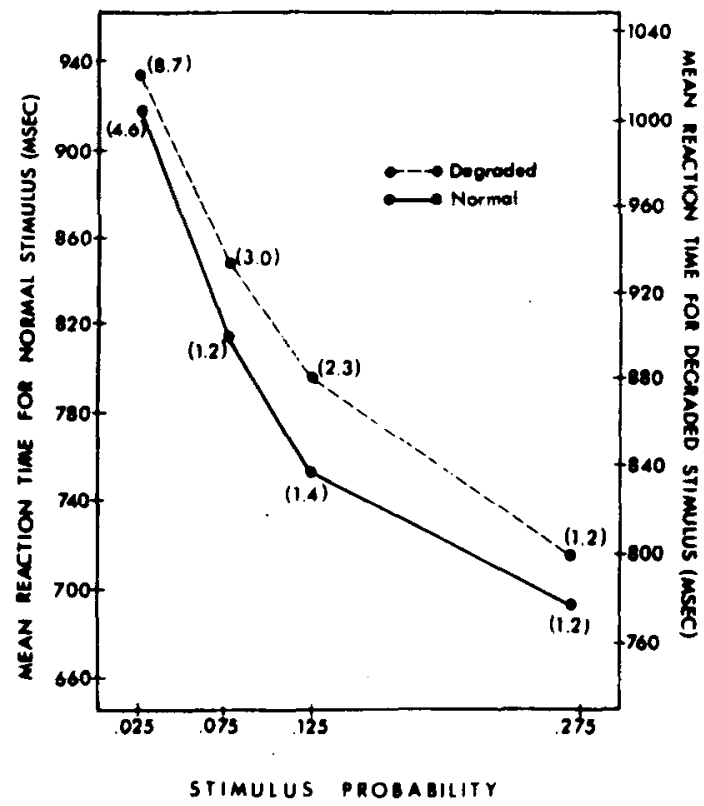

Figure 3. Reaction time as a function of stimulus probability for the normal stimulus and degraded stimulus conditions in Experiment II. (The average percentage error at each point is indicated in parentheses. The scale for the normal stimulus condition has been shifted upwards.) 


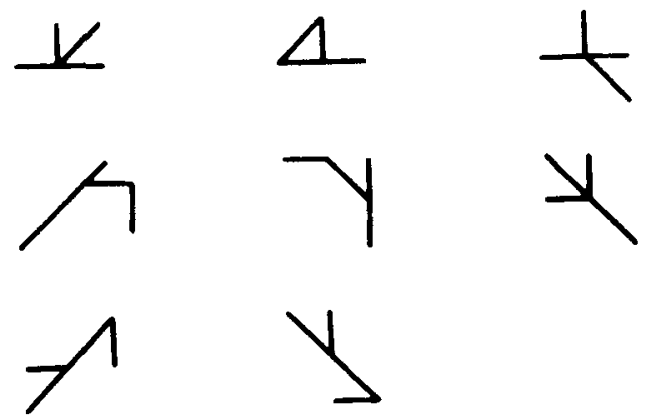

Figure 4. Stimuli used in Experiment III.

Figure 5. In the analysis of error rates, the only significant main effects were those of Practice $[F(1,22)=15.7$, $\left.\mathrm{p}<.01, \mathrm{MS}_{\mathrm{e}}=24,768\right]$ and Probability $[\mathrm{F}(3,66)=$ 22.6, $\left.\mathrm{p}<.01, \mathrm{MS}_{\mathrm{e}}=18,687\right]$. The interaction of Practice by Order of Degraded vs. Normal Blocks was significant $\left[F(1,22)=4.36, p<.05, \mathrm{MS}_{\mathrm{e}}=24,768\right]$, with the group receiving the Normal block first improving more between the first and last two blocks than the group receiving the Degraded block first. Also significant was the Order by Quality interaction $\left[\mathrm{F}(1,22)=7.5, \mathrm{p}<.05, \mathrm{MS}_{\mathrm{e}}=7,329\right]$, with the Quality effect being larger for the group starting with a degraded block. The Quality by Practice interaction was also significant $\left[\mathrm{F}(1,22)=5.48, \mathrm{p}<.05, \mathrm{MS}_{\mathrm{e}}=6,314\right]$, with the effect of Quality decreasing between the first and last two blocks. In the analysis of RTs, the main effects of Practice $\left[\mathrm{F}(1,22)=38.7, \mathrm{p}<.01, \mathrm{MS}_{\mathrm{e}}=\right.$ $60,050]$, Quality $\left[\mathrm{F}(1,22)=10.4, \mathrm{p}<.01, \mathrm{MS}_{\mathrm{e}}=\right.$ $67,940]$, and Probability $[F(3,66)=23.8, p<.01$, $\mathrm{MS}_{\mathrm{e}}=44,785$ ] were significant. The only significant two-way interaction was that of Order by Quality $\left[F(1,22)=19.2, \quad \mathrm{p}<.01, \mathrm{MS}_{\mathrm{e}}=67,940\right]$, with the Quality effect larger for the group starting with a degraded block, as in the error rate data. The interaction of particular interest, that of Quality by Probability, shown in Figure 5, was particularly small $[\mathrm{F}(3,66)=$ $\left..09, \mathrm{MS}_{\mathrm{e}}=18,771\right]$.

\section{GENERAL DISCUSSION}

The results of these three experiments indicate that two distinct encoding processes are used in character classification tasks. The results of Experiment I, taken together with the results of Miller and Pachella (1973) show that, when digits are used as stimuli, the encoding process is affected by probability. When the stimuli are nonsense shapes or forms, however, the encoding process is not affected by probability, as demonstrated in Experiments II and III. Thus, these data provide strong evidence that the encoding process operates in a fundamentally different manner when it is dealing with digits as opposed to nonsense forms. One obvious difference between the stimulus materials is that verbal codes are readily available in the case of digits, whereas the nonsense forms did not have readily available verbal codes. For both types of stimuli, the encoding mech. anism must first internalize the probe on the basis of its visual features. For alphanumeric stimuli it appears that the next step is for the encoding process to retrieve the name of the probe for use in the comparison process, while for nonsense forms this may not be done. Thus, one explanation for the interaction observed in Experiment $I$ is that probability and quality both affect the process of retrieving the name of a probe. If this simple translation is not made in the case of nonsense forms, there would be no interaction of probability and quality when they were used as stimuli. Converging operations are needed before this explanation could be accepted as more than a tentative hypothesis, sirce it is possible that subjects constructed and used their own codes for the nonsense forms used in Experiment II and III. Such name codes would be very different from the highly overlearned names associated with letters or digits, however,

Another possible explanation is that the crucial difference in stimulus materials was that the digits were familiar, while the forms were unfamiliar. It could be argued that probability affects encoding of familiar stimuli but not unfamiliar stimuli. This argument is not supported by other research (Pachella \& Miller, 1976) in which probability was not found to affect encoding of letters in a letter-matching task.

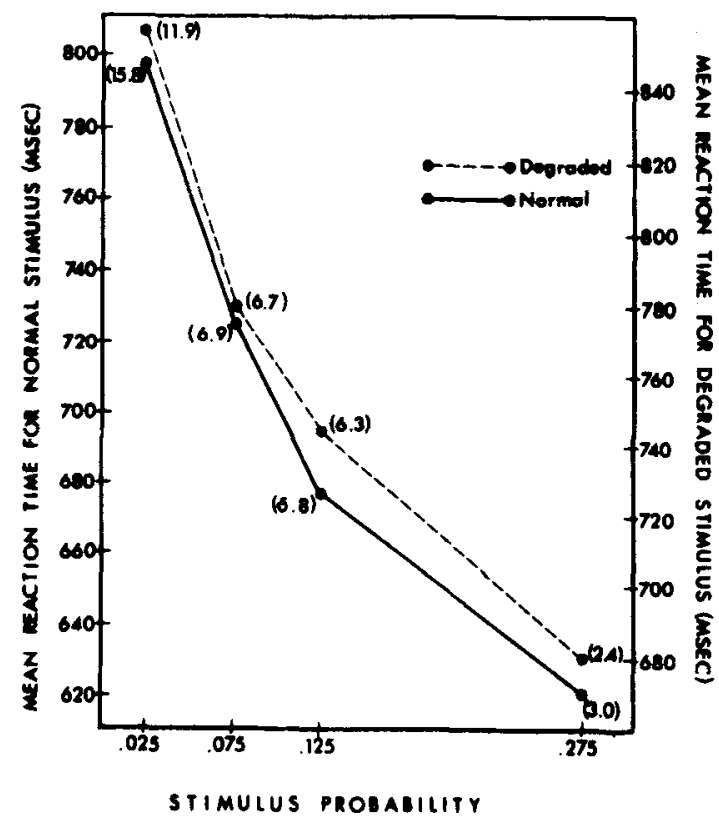

Figure 5. Reaction time as a function of stimulus probability for the normal stimulus and degraded stimulus conditions in Experiment III. (The average percentage error at each point is indicated in parentheses. The scale for the normal stimulus condition has been shifted upwards.) 
One objection that might be raised to the interpretation of these data is that stimulus quality might affect some stage other than the encoding stage. Sternberg (1967) found evidence that quality affects the comparison stage, though only for relatively unpracticed subjects. Thus, it might be argued that probability and quality interact because both affect the comparison stage. This argument seems particularly implausible because of the lack of interaction in Experiments II and III, however. The idea that visual aspects of stimulus quality affect the comparison stage seems to indicate that the internal representation is a visual one (Sternberg, 1967). Thus, one would expect at least as large a Probability by Quality interaction in tasks where subjects are more likely to use a visual code, such as Experiments II and III. The lack of interaction in these two experiments is evidence against the argument that probability and quality interact because both affect the comparison stage.

Though probability did not seem to affect the encoding stage in the experiments with nonsense forms, it definitely did have a sizeable effect. This result is problematic for the memory scanning model of Sternberg (1969), since this model can best explain probability effects in the encoding stage. The result is not inconsistent with the self-terminating scan model of Theios et al. (1973), however. In this model, probability is assumed to affect the order of comparison in a selfterminating comparison stage. Since subjects search memory with a visual representation of the probed nonsense form, this model would have to assume that the encoding stage would remove all effects of degradation initially, otherwise a Probability by Quality interaction would be found. Furthermore, the time that the preprocessing mechanism takes to remove degradation would have to be independent of stimulus probability. Only under these conditions could the probability effect have arisen through the comparison stage, as in the model of Theios et al. (1973), without producing an interaction of quality and probability.

Regardless of the suitability of the present suggestion, future attempts to produce theoretical accounts of memory scanning will have to consider the nature of the material to be dealt with by the subject. The current models tend to describe sequences of processes that could potentially be operative on any form of stimulus input. The present experiments demonstrate that relationships between previously considered variables (degradation and probability) are modified by the nature of the stimulus materials. Thus, general processing schemes must take into account this factor.

\section{REFERENCE NOTES}

1. Wattenbarger. B. L. The representation of the stimulus in charucter classification. Unpublished doctoral dissertation. University of Michigan, 1970.

2. Nickerson, R. S. Binary-classification reaction time: A revien of some studies of human information-processing capabilities(Report No. 2004). Cambridge. Mass: Bolt. Beranek and Newman. 1970.

\section{REFERENCES}

Biederman, I.. \& Stacy, E. W. Stimulus probability and stimulus set size in memory scanning. Joumal of Experimental Psychology. 1974, 102. 1100.1107.

BraceY, G. W. Two operations in character recognition: A partial replication. Perception \& Psychophysics. 1969. 6. 357-360.

Briggs, G. E.. \& Swanson. J. M. Encoding. decoding. and central functions in human information processing. Journal of Experimental Psychology. 1970. 86. 296-308.

Chase, W., \& Calfee, R. G. Modality and similarity effects in short-term recognition memory. Joumal of Experimental Psychology. 1969. 81, 510-514.

Klatzky, R. L., \& Sмith, E. E. Stimulus expectancy and retrieval from short-term memory. Joumal of Experimental Psychology, 1972, 94, 101-107.

Miller, J. O.. \& Pachella, R. G. Locus of the stimulus probability effect. Journal of Experimental Psychology. 1973, 101. 227.231.

Pachella, R. G.. \& Miller, J. O. Stimulus probability and same-different classification. Perception \& Psychophysics. 1976. 19. 29.34.

Sternberg, S. High speed scanning in human memory. Science. $1966,153,652-654$.

Sternberg, $S$. Two operations in character recognition: Some evidence from reaction-time measurements. Perception \& Psychophysics, 1967, 2, 45-53.

Sternberg. S. Memory scanning: Mental processes revealed by reaction time experiments. American Scientist, 1969. 57. $421-457$.

ThEIOS, J. The components of response latency in simple human information processing tasks. In P. M. A. Rabbitt \& S. Dornic (Eds.). Attention and performance, V. New York: Academic Press, 1975.

Theios, J., Smith, P. G., Haviland, S. E., Traupmann, J., \& MoY. M. C. Memory scanning as a serial, self-terminating process. Journal of Experimental Psychology, 1973, 97, 323-336.

Theios, J., \& WALTER, D. G. Stimulus and response frequency and sequential effects in memory scanning reaction times. Journal of Experimental Psychology, 1974, 102, 1092-1099.

\section{NOTE}

1. The use of "name code" here and elsewhere in this paper refers to an identifying code whose relations to the stimulus is very well established in long-term memory. This usage is consistent with previous usage, particularly by Theios (1975).

(Received for publication June 23, 1975; revision accepted December 19, 1975.) 